\title{
Procesos pterigoideos y la sincondrosis esfeno-occipital en la disyunción palatina
}

\author{
Pterygoid processes and synchondrosis spheno-occipital in the Rapid \\ Maxillary Expansión
}

\section{Resumen}

La disyunción palatina que consiste en la apertura de la sutura palatina media, es usada frecuentemente para corregir la arcada maxilar estrecha transversalmente y ya que el maxilar se relaciona también con la base del cráneo el objetivo de este estudio fue revisar en la literatura los efectos que la disyunción palatina produce sobre los procesos pterigoideos y la sincondrosis esfeno-occipital, analizados clínicamente, con tomografías computarizadas, gammagrafía ósea, radiografías cefalométricas y el método de elementos finitos. La literatura revisada fue hecha sobre las bases de datos: Medline (PubMed), Scopus, Scielo, BSV (Bireme), ISI (Web of Sciencie) y Lilacs. Se puede afirmar que la fuerza proporcionada durante una disyunción palatina no solo actúa al nivel de la sutura palatina media, sino también sobre los procesos pterigoideos y hasta sobre la sincondrosis esfeno-occipital, ocasionando desplazamientos y diferentes concentraciones de tensiones.

Palabras clave: Disyunción palatina; base de cráneo; sincondrosis esfeno-occipital.

\begin{abstract}
The Rapid Maxillary Expansion that involves the opening of the median palatine suture is used frequently to correct narrow arch maxillary and since the maxillary relates also with the skull base, the objective of this paper was to search in the literature the effects that the Rapid Maxillary Expansion produces on the pterygoid process and the spheno-occipital synchondrosis; analyzed clinically, with compute tomography, bone scintigraphy, cephalometric X-ray and the finite element method. The literature review was done on the data bases: Medline (PubMed), Scopus, Scielo, BSV (Bireme), ISI (Web of Sciencie) and Lilacs. It can be said that the forces it proportionate during the Rapid Maxillary Expansion not only to performs on the median palatine suture, but also on the pterygoid processes and even on the spheno-occipital synchondrosis, to making displacements and different concentrations of stress. Keywords; Rapid Maxillary Expansion; Skull base; Spheno-occipital synchondrosis.
\end{abstract}

\section{Introducción}

La disyunción palatina es usada frecuentemente para corregir la atresia transversal maxilar, la mordida cruzada posterior y el aumento del perímetro del arco dentario. Estando esta fuerza proporcionada por el aparato disyuntor ortodóntico concentrada inicialmente sobre el ancho del paladar, también desplazamientos simultáneos aparecen en las suturas circunmaxilares $^{1,2}$ (Fig. 1). Algunos estudios sobre los efectos de la disyunción palatina sobre la base de cráneo y la sincondrosis esfeno-occipital en niños mostraron una apertura y un significativo aumento en la actividad metabólica de esta zona. Estas referencias sumadas a la evidencia de que la proliferación condrocítica es modulada por la tensión mecánica, sugiere preguntas sobre la influencia de la disyunción palatina sobre el cartílago de la sincondrosis esfenooccipital en la base del cráneo ${ }^{3,4 .}$

La base del cráneo se localiza entre el neurocráneo y el tercio medio de la cara; sus huesos en personas en creci- miento están unidos por suturas tipo sincondrosis, que constituyentes de cartílago permitirían cierta flexibilidad y desplazamientos cuando presiones o tensiones mecánicas son ejercidas sobre ellas 5 (Fig. 2). Se sabe también que las fuerzas transversales de la disyunción palatina son transmitidas hasta la base de cráneo por la unión entre la tuberosidad del maxilar y los procesos pterigoides del hueso esfenoide. La extensión de los efectos de este procedimiento ortodóntico sobre la base del cráneo aún no han sido extensamente estudiados ni bien determinados ${ }^{6,7}$.

En ese sentido, el objetivo del estudio fue revisar en la literatura los efectos biomecánicos que la disyunción palatina produce sobre los procesos pterigoideos del hueso esfenoide y la sincondrosis esfeno-occipital en la base del cráneo.

\section{Materiales y Método}

La literatura fue revisada sobre la siguientes bases de datos: Medline (PubMed), Scopus, Scielo, BSV (Bireme),
Manuel Chávez-Sevillano ${ }^{1}$, Ana Cláudia Rossi $^{2}$, Alexandre Freire ${ }^{2}$, Carlos Carranza², Jessica Arieta Miranda1, Felippe Bevilacqua Prado²

1.Departamento Académico de Estomatología Pediátrica de la Facultad de Odontología de la Universidad Nacional Mayor de San Marcos.

2.Departamento de Morfología de la Facultad de Odontología de Piracicaba de la Universidad Estadual de Campinas.

Correspondencia:

Mg. Esp. Manuel Chávez-Sevillano

Av. José Santos Chocano 1270.

Las Palmeras. Lima 39, Perú.

Email: mchavezs@unmsm.edu.pe

Coautores

Rossi: anaanatomia@gmail.com

Freire: alerfreire@gmail.com

Carranza: odontocarlos

Arieta: jam-299@hotmail.com

Bevilacqua:fprado@fop.unicamp.br

Recibido: 20-05-14

Aceptado: 12-05-15
ISI (Web of Sciencie) y Lilacs, usando las palabras claves: Disyunción palatina, sincondrosis esfeno-occipital y base de cráneo. Los manuscritos y libros evaluados fueron publicados de 1961 hasta 2014. Fueron encontrados 45 artículos, de los cuales se utilizaron solo 20 por tener mayor relevancia para el tema de revisión.

\section{Revisión de Literatura}

Apertura, tensiones y desplazamientos de la sincondrosis esfeno-occipital durante la disyunción palatina.

En el estudio realizado en primates Rhesus, Gardner \& Kronman ${ }^{8}$ mostraron que algunas suturas de la base del cráneo podían ser abiertas durante una disyunción palatina. Una apertura de $1.5 \mathrm{~mm}$. de la sincondrosis esfenooccipital (SE) fue encontrada en el experimento animal. Holberg', usando valores de distintas propiedades mecánicas (Módulo de Young y Coeficiente de Poisson), para simular virtualmente al hueso joven y hueso adulto, realizó 
un estudio con el Método de Elementos Finitos (MEF). Simuló una disyunción palatina e hizo una flexión lateral de los procesos pterigoides del esfenoide de 1 , 2, 3, 4 y $5 \mathrm{~mm}$. Para la flexión de $2 \mathrm{~mm}$ encontró valores de apertura de la sincondrosis esfeno-occipital de $0.93 \mathrm{~mm}$. para el hueso joven y $0.95 \mathrm{~mm}$ para el hueso adulto. En lo que respecta a las tensiones, en la sincondrosis esfenooccipital se encontraron valores de 1.6 Megapascales (Mpa.) para el hueso joven, mientras que para el adulto 9.0 Mpa. Holberg \& Rudzki - Janson ${ }^{10}$, utilizaron los datos tomográficos de dos cráneos secos de adulto y joven para simular por el MEF la distribución tensiones y desplazamientos de estructuras anatómicas durante una apertura lateral simulada de los procesos pterigoideos de $2.5 \mathrm{~mm}$. Las tensiones encontradas sobre la sincondrosis esfeno-occipital fueron de 38.8 Mpa y 21.2 Mpa para los cráneos adulto y joven respectivamente. Holberg y cols. ${ }^{11}$ realizaron 4 modelos de cráneos convertidos en modelos de elementos finitos para analizar la reducción de tensiones durante una disyunción palatina cuando aplicaban diferentes incisiones quirúrgicas. Con una apertura de la sutura media palatina de $5 \mathrm{~mm}$. la concentración de tensiones en la SE fueron: modelo sin cirugia: $12.8 \mathrm{Mpa}$, modelo con corticotomia zigomaticomaxilar: $12.9 \mathrm{Mpa}$, modelo con corticotomia lateral completa: 8.9 Mpa y modelo con Lefort I: 3.8 Mpa (Fig. 3).

En otro estudio utilizando el MEF, Gautam y cols. ${ }^{12}$ simularon una disyunción palatina utilizando sulfato de bario en estructuras anatómicas de un cráneo seco de 7 años de edad. Se identificó un desplazamiento lateral de la SE de $0.000000000041 \mathrm{~mm}$. Leonardi y cols. ${ }^{4}$ realizaron un estudio tomográfico en 8 pacientes en crecimiento. Antes de la disyunción palatina la medida promedio sagital de la SE fue de $1.73 \mathrm{~mm}$ +/- 0.46mm. Al terminar la disyunción palatina esta medida fue de $2.30 \mathrm{~mm}$ $+/-0.47 \mathrm{~mm}$, con una diferencia estadísticamente significativa. Por lo tanto, concluyeron que la disyunción palatina ocasionaba una pequeña apertura anteroposterior de la sincondrosis esfenooccipital en jóvenes.

Silvestrini-Biavati y cols. ${ }^{13}$ evaluaron los cambios en la SE hasta un año después de realizar la disyunción palatina. Fueron registradas radiografías cefalométricas de dos grupos. Grupo 1 (experimental) que tuvo 30 pacientes, 13 masculinos y 17 femeninos con edad promedio de $8+/$ - 1.5 años. Estos re- cibieron tratamiento de disyunción palatina con el aparato de Hass. El Grupo 2 (control) incluyó 14 personas no tratadas, 6 masculinos y 8 femeninos con edad promedio de $7.6+/-1.7$ años. Medidas cefalométricas identificando la sincondrosis esfeno-occipital fueron identificadas antes y después de la disyunción palatina: SOS-Ba (sincondrosis esfeno-occipital-Basión) y SOS-S (sincondrosis esfeno-occipitalSella). Una apertura estadísticamente significativa de la sincondrosis esfenooccipital y un aumento de la longitud de la base posterior del cráneo fue encontrado en ambos grupos después de la disyunción palatina. Sin embargo, estos cambios no afectaron las medidas verticales $\mathrm{u}$ horizontales del complejo máxilo mandibular.

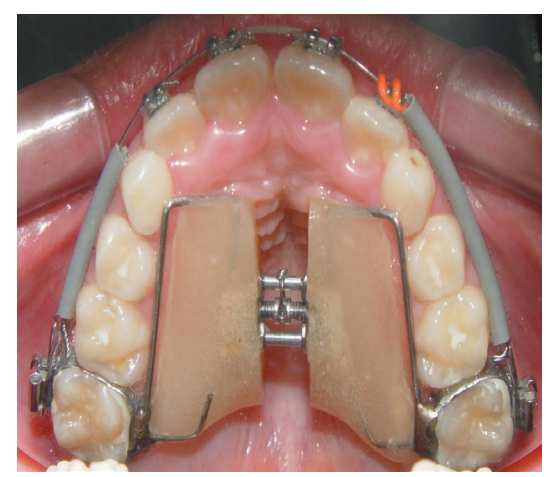

Fig. 1. Disyunsión palatina

Fuente: Autor

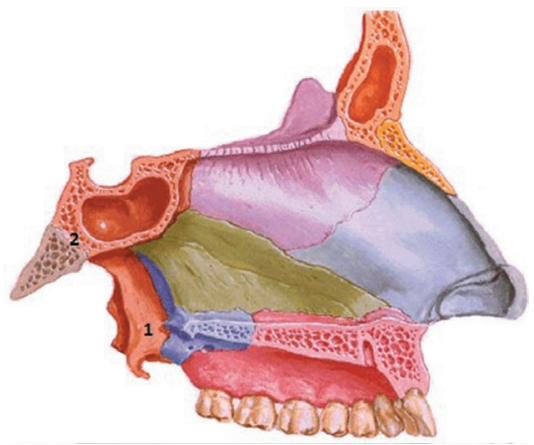

Fig. 2. Relación anatómica entre el maxilar, el proceso pterigoide (1) y la sincondrosis esfenooccipital(2).

Fuente: Modificado de Sobota, 2006.

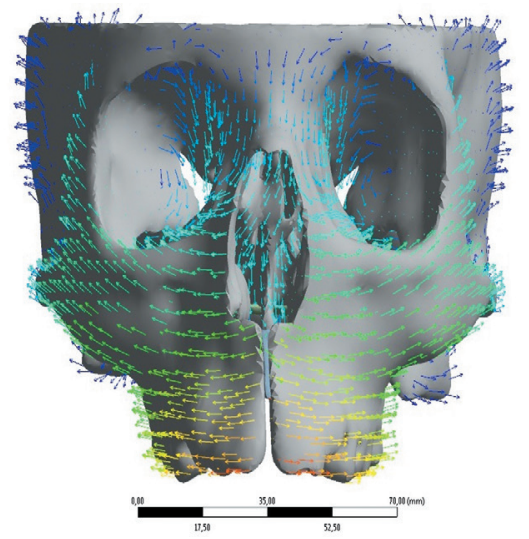

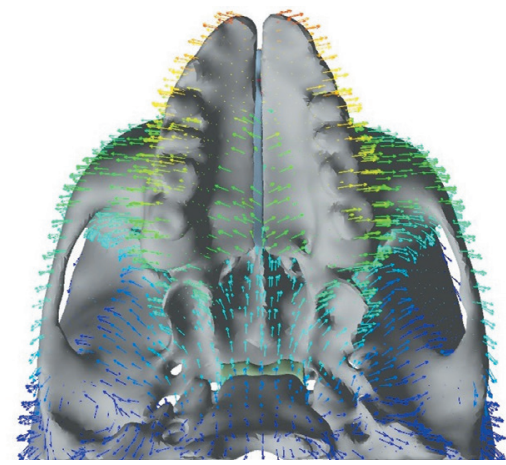

Fig. 3. Vista frontal e inferior de un cráneo humano simulando una disyunción palatina por el Método de Elementos Finitos.

Fuente: Laboratorio de Elementos Finitos- FOPUNICAMP.

Metabolismo de la sincondrosis esfenooccipital durante la disyunción palatina

Baydas y cols. ${ }^{3}$ evaluaron con cintigrafía ósea, los efectos esqueléticos en una disyunción palatina sobre as estructuras craneofaciales. Fueron 17 pacientes con intervalo de edad entre 16.1 hasta 18.1 años. Fueron registradas con cintigrafía ósea antes (T1), durante (T2) y después de la fase activa (T3) de la disyunción palatina. Encontraron un aumento significativo del metabolismo óseo en la sincondrosis esfeno-occipital durante la interfase T1-T2. Concluyeron que la disyunción palatina no solo tiene efectos dentales si no también efectos esqueléticos en pacientes jóvenes.

\section{Tensiones y desplazamientos laterales de los procesos pterigoideos durante la disyunción palatina}

Timms ${ }^{14}$ fue uno de los primeros en analizar los efectos de la disyunción palatina sobre estructuras esqueléticas y no solo dentales. En 32 pacientes con edades entre 8.2 años hasta 24.1 ańos, recibieron el tratamiento de disyunción palatina y fueron evaluados clínicamente identificando la distancia transversal del hámulo pterigoideo, el cual se localiza en la región inferior de la lámina medial de los procesos pterigoideos del hueso esfenoide. Los resultados mostraron un incremento de la distancia inter hamular desde $3 \mathrm{~mm}$ hasta $7 \mathrm{~mm}$. Iseri y cols. ${ }^{15}$, estudiaron el cráneo de un niño de 12 años de edad, con mordida cruzada posterior y paladar estrecho. Aplicando el MEF simularon una apertura de $5 \mathrm{~mm}$ de la sutura palatina media. La parte inferior del proceso pterigoideo se desplazó $4.9 \mathrm{~mm}$ lateralmente y tuvo una concentración de tensión de 6.20 $\mathrm{Kg} / \mathrm{mm}^{2}$. La parte media del proceso pterigoideo se desplazó $4.8 \mathrm{~mm}$ y tuvo una concentración de tensión de 26.95 $\mathrm{Kg} / \mathrm{mm}^{2}$. Finalmente la parte superior cercana a la base del cráneo tuvo un 
desplazamiento lateral de $1.4 \mathrm{~mm}$ con una concentración de tensión de 73.75 $\mathrm{Kg} / \mathrm{mm}^{2}$.

Jafari y cols. ${ }^{6}$ de las imágenes tomográficas de un cráneo seco de aproximadamente 12 años de edad, hizo un estudio por el MEF simulando una disyunción palatina. Identificó también desplazamientos laterales en los procesos pterigoideos. En la parte superior con valores de $0.21 \mathrm{~mm}$ para a lámina medial y $0.44 \mathrm{~mm}$ para la lámina lateral. Sin embargo, el desplazamiento en la parte inferior del proceso pterigoideo fue mayor con valores de $1.86 \mathrm{~mm}$ y $2.07 \mathrm{~mm}$ para as láminas medial y lateral respetivamente. Holberg \& Rudzki-Janson ${ }^{10}$, simularon una disyunción palatina en dos cráneos, de joven y adulto. Simularon una flexión lateral de los procesos pterigoideos de $2.5 \mathrm{~mm}$ encontrando distintos valores de tensiones; siendo para el cráneo joven: en la lámina lateral del proceso pterigoideo 87.1 Mpa y en la lámina medial $169.9 \mathrm{Mpa}$. Sin embargo en el cráneo de adulto: en la lámina lateral del proceso pterigoideo 366.8 Mpa y en la lámina medial 236.6 Mpa.

Pan y cols ${ }^{16}$, exploraron los efectos biomecánicos de la disyunción palatina sobre un cráneo de un paciente de 14 ańos con paladar fisurado unilateral por medio del MEF. Simularon una apertura de la sutura palatina mediana de $5 \mathrm{~mm}$. La parte superior del proceso pterigoideo cerca de la base del cráneo mostro un mínimo desplazamiento lateral siendo los valores de $0.68 \mathrm{~mm}$ para la lámina medial y $0.62 \mathrm{~mm}$ para la lámina lateral del lado no fisurado. Sin embargo, la parte inferior mostró un mayor desplazamiento lateral siendo de $1.41 \mathrm{~mm}$ y $1.53 \mathrm{~mm}$ para las láminas lateral y medial respetivamente. Holberg y cols. ${ }^{11}$, hicieron también la reproducción de 4 modelos de cráneos completos por el MEF para analizar la reducción de la tensión durante la disyunción palatina cuando aplicaban diferentes incisiones quirúrgicas. Con una abertura de la sutura palatina media de $5 \mathrm{~mm}$, la lámina medial del proceso pterigoideo tuvo las siguientes concentraciones de tensiones: modelo sin cirugía 205.9 Mpa, modelo con corticotomía cigomático-maxilar $179.7 \mathrm{Mpa}$, modelo con corticotomía lateral completa 176.9 Mpa y en el modelo con Lefort I 114.4 Mpa. Por otro lado, en la lámina lateral de los procesos pterigoideos tuvieron la siguiente concentración de tensiones: modelo sin cirugía $180 \mathrm{Mpa}$, modelo con corticotomía cigomaticomaxilar 178.2 Mpa, modelo con corti- cotomía lateral completa 136.5 Mpa y en el modelo con Lefort I 109.9 Mpa.

Gautam y cols. ${ }^{12}$, analizaron por el MEF y sulfato de bario, un cráneo seco de edad aproximada de 7 años. Después de simular una disyunción palatina encontraron desplazamientos laterales en los procesos pterigoideos. En la lámina lateral, en la zona superior fue $0.84 \mathrm{~mm}$ e inferior fue 1.26, sin embargo en la lámina medial zona superior fue $0.9 \mathrm{~mm}$ e inferior $1.36 \mathrm{~mm}$.

Boryor y cols. ${ }^{17}$, simularon una disyunción palatina en un cráneo seco de edad 35 años y por el MEF determinaron el desplazamiento lateral de los procesos pterigoideos cuando aplicaron una fuerza de 100 Newton. Como resultados encontraron que el desplazamiento de la lámina medial fue de $1.53 \mathrm{~mm} \mathrm{y}$ de la lámina lateral fue de $1.23 \mathrm{~mm}$; mientras que el desplazamiento del hámulo pterigoideo fue de $1.31 \mathrm{~mm}$. Baldawa $\&$ Bhad $^{18}$, en otro similar estudio hecho sobre el cráneo seco de aproximadamente 20 años de edad, después de simular una abertura de $5 \mathrm{~mm}$ de la sutura palatina por medio del MEF, encontraron concentración de tensiones y desplazamientos en el plano transversal de los procesos pterigoideos. Los desplazamientos y tensiones encontrados en la lámina medial pterigoidea, en la parte superior fueron de $1.8 \mathrm{~mm}$ y $74,24 \mathrm{~kg} /$ $\mathrm{mm} 2$ respectivamente; mientas que en la parte inferior fueron de $2.04 \mathrm{~mm}$ y $14.24 \mathrm{~kg} / \mathrm{mm}^{2}$. Sin embargo, en la lámina lateral, la parte superior mostró un desplazamiento de $0.32 \mathrm{~mm}$ y $68.17 \mathrm{~kg} / \mathrm{mm}^{2}$ de tensión, mientras que la parte inferior se desplazó $2.34 \mathrm{~mm}$ y concentró $12.24 \mathrm{~kg} / \mathrm{mm}^{2}$ de tensión.

Provatidis y cols. ${ }^{19}$, hizo un estudio en un cráneo seco por el MEF y simuló una disyunción palatina. Los resultados fueron comparados con otro estudio clínico y un estudio in vitro del mismo cráneo. Afirmó que la sutura entre el proceso pterigoideo y el maxilar no influencia en el resultado de la disyunción maxilar.

Wang y cols ${ }^{20}$, también hicieron un estudio con el MEF en un cráneo de un paciente con paladar fisurado de 14 años de edad. Simularon una disyunción palatina de $5 \mathrm{~mm}$, encontrando un desplazamiento lateral de los procesos pterigoideos. Los resultados en el lado no fisurado, en la parte superior de la lámina medial fue de $1.38 \mathrm{~mm}$ y en la lámina lateral $1.37 \mathrm{~mm}$. Mayor desplazamiento se encontró en la parte inferior de los procesos siendo de $2.95 \mathrm{~mm}$ y $2.99 \mathrm{~mm}$ en las láminas medial y lateral respetivamente.

Gautam y cols ${ }^{21}$,de las imágenes tomográficas de un paciente con paladar fisurado de 16 años, realizaron un estudio con el MEF simulando también una disyunción palatina. En este caso los desplazamientos y tensiones encontradas en el proceso pterigoideo del lado no fisurado fueron: lámina lateral, en la parte superior $0.82 \mathrm{~mm}$ de desplazamiento y $11.2 \mathrm{~N} / \mathrm{mm}^{2}$ de tensión, y en la parte inferior $1.08 \mathrm{~mm}$ de desplazamiento y $5.7 \mathrm{~N} / \mathrm{mm}^{2}$ de tensión. Para la lámina medial parte superior fue $0.42 \mathrm{~mm}$ de desplazamiento y $418.6 \mathrm{~N} /$ $\mathrm{mm}^{2}$ de tensión y en la parte inferior $1.11 \mathrm{~mm}$ de desplazamiento y $23.1 \mathrm{~N} /$ $\mathrm{mm}^{2}$ de tensión. Los mismos autores, en el mismo modelo de cráneo y en otro estudio con MEF simularon y evaluaron los desplazamientos laterales y las tensiones que aparecen en los procesos pterigoideos durante una disyunción palatina con distintas técnicas de ayuda quirúrgicas $^{22}$. Con la técnica de osteotomía del pilar cigomático y disyunción pterigopalatina los desplazamientos y tensiones en el lado no fisurado fueron: en la lámina medial parte superior $0.25 \mathrm{~mm}$ y $105.3 \mathrm{~N} / \mathrm{mm} 2$ respectivamente; mientras que en la parte inferior fue de $0.97 \mathrm{~mm}$ y $9.7 \mathrm{~N} / \mathrm{mm} 2$ respectivamente. Sin embargo, en la lámina lateral, parte superior fue de $0,32 \mathrm{~mm}$ y $47.8 \mathrm{~N} / \mathrm{mm}^{2}$ respectivamente, mientras que la parte inferior obtuvieron los valores de $0.88 \mathrm{~mm}$ y $7.5 \mathrm{~N} / \mathrm{mm}^{2}$ respectivamente. Con la técnica de osteotomía de la sutura palatina media, los desplazamientos y las tensiones en el lado no fisurado fueron: en la lámina medial parte superior fueron de $0.63 \mathrm{~mm} \mathrm{y}$ $33.3 \mathrm{~N} / \mathrm{mm}^{2}$ respectivamente, mientras que en la parte inferior fue de $1.02 \mathrm{~mm}$ y $1.5 \mathrm{~N} / \mathrm{mm}^{2}$ respectivamente. Sin embargo, en la lámina lateral, parte superior se encontraron valores de $0.28 \mathrm{~mm}$ y $29.3 \mathrm{~N} / \mathrm{mm}^{2}$ respectivamente, mientras que en la parte inferior fue de $0.92 \mathrm{~mm}$ y $29.3 \mathrm{~N} / \mathrm{mm}^{2}$ respectivamente. Con la técnica de osteotomía tipo Lefort I, disyunción pterigopalatina y osteotomía de la sutura palatina media, los desplazamientos y tensiones en el lado no fisurado fueron: en la lámina medial parte superior fue de $0.52 \mathrm{~mm}$ y $43.0 \mathrm{~N} / \mathrm{mm}^{2}$ respectivamente, mientras que en la parte inferior fue de $1.08 \mathrm{~mm}$ y $1.9 \mathrm{~N} / \mathrm{mm}^{2}$ respectivamente. Sin embargo, en la lámina lateral, parte superior encontraron valores de $0.34 \mathrm{~mm}$ de desplazamiento y $30.4 \mathrm{~N} / \mathrm{mm}^{2}$ de tensión, mientras que la parte inferior mostro valores de $0.98 \mathrm{~mm}$ de desplazamiento y $4.2 \mathrm{~N} / \mathrm{mm}^{2}$ de tensión. 
Lagravere y cols. ${ }^{23}$ realizaron un estudio prospectivo tomográfico para comparar los efectos dentales y esqueléticos de dos aparatos disyuntores, el aparato hyrax convencional y un disyuntor con anclaje esquelético dado por mini implantes ortodónticos. La muestra fue constituida por 62 pacientes adolescentes dividida en tres grupos incluyendo un grupo control.

A nivel de los procesos pterigoideos no fueron encontrados ningún cambio fue encontrado entre los grupos experimentales y el grupo control.

\section{Conclusiones}

En la mayoría de los estudios anteriormente descritos indican que la fuerza proporcionada durante una disyunción palatina, no actúan aisladamente al nivel de la sutura palatina media, sino también sobre los procesos pterigoideos del hueso esfenoide y la sincondrosis esfeno-occipital en la base del cráneo. Esta fuerza sería responsable de desplazamientos y concentración de tensiones en dichas delicadas estructuras anatómicas; siendo esto información útil en la comprensión de los efectos biológicos y mecánicos de la disyunción palatina que debe ser considerado por el clínico durante su terapéutica.

\section{Referencias bibliográficas}

1. Hass AJ. Rapid expansion of the maxillary dental arch and nasal cavity by opening the midpalatal suture. Angle Orthod. 1961;31:73-90.

2. Garret BJ, Caruso JM, Rungcharassaeng K, Farrage JR, Kim JS, Taylor GD. Skeletal effects to the maxila after rapid maxillary expansion assessed with cone-beam computed tomography. Am. J. Orthod Dentofacial Orthop. 2008;134:8-9.

3. Baydas B, Yavuz I, Uslu H, Dagsuyu I.M, Ceylan I. Nonsurgical rapid maxillary expansion effects on craniofacial structures in Young adult females. Angle Orthod.2006;76:759-767.

4. Leonardi R, Cutrera A, Barbato E. Rapid maxillary expansion affects the spheno-occipital synchondrosis in youngsters. Angle Orthod. 2010;80:106-110.

5. Sato S. A Treatment Approach to Malocclusion Under the Consideration of Craniofacial Dynamics. Kanawaga Dental Collegue, Japon 1991.
6. Jafari A, Shetty KS, Kumar M. Study of stress distribution and displacement of various craniofacial structures following application of transverse orthopedic forces- a three dimensional FEM study. Angle Orthod. 2003;73:1220.

7. Provatidis C, Georgiopoulos B, Kotinas A and McDonald J P. In vitro validate finite element method model for a human skull and related craniofacial effects during rapid maxillary expansion. Journal Engineering in Medicine. Proc. I MechE (220) Part H, 2006.

8. Gardner Gerald E, Kronman Joseph H. Cranioskeletal displacements caused by rapid palatal expansion in the rhesus monkey. American J. Orthodontic. 1971;51(2)

9. Holberg C. Effects of Rapid Maxillary Expansion on the Cranial Base- an FEM -Analysis. J.Orofac. Orthop. 2005;66:54-66.

10. Holberg Chistof, Rudzki-Janson Ingrid. Stress at the Cranial Base Induced by Rapid Maxillary Expansion. Angle Orthodontist. 2006:76(4)

11. Holberg C, Steinnhauser S, Rudzki-Janson I. Rapid maxillary expansión in adults: cranial stress reduction depending on the extend of surgery. Eur J Ortho 2007;29:31-6.

12. Gautam Pawam, Valiathan Ashima, Adhikari Raviraj. Stres and displacement patterns in the craniofacial skeleton with rapid maxillary expansion: A finite element method study. Am. J. Orthod. Dentofacial Orthop 2007;132:e15.e11.

13. Silvestrini-Biavati A, Angiero F, Gambino A, Ugolini A. Do changes spheno-occipital synchondrosis after rapid maxillary exoansion affect the maxillomandibular complex?. European Journal of Pediatric Dentistry. 2013;14(1)

14. Timms DJ. A study of basal movement with rapid maxillary expansion. Am. J. Orthod. 1980 may;77(5).

15. Iseri H, Tekkaya AE, Oztan O, et al. Biomechanical effects of rapid maxillary expansión the craniofacial skeleton, studied by the finite element method. Eur J Orthod. 1998;20:247-56
16. Pan Xiaogang, Quian Yufen, Yu Jia, Wang Dongmei, Tang Yousheng and Shen Gang. Biomechanical Effects of Rapid Palatal Expansion on the Craniofacial Skeleton Wtth Cleft Palate: A three-Dimensional Finite Element Analysis. Cleft Palate-Craniofacial Journal, March 2007;44(2)

17. Boryor Andrew, Martin Geiger, Ansgar Hohmann, Arthur Wunderlich, Christian Sander, Franz Martin Sander, Franz Gunter Sander. Stress distribution and displacement analysis during an intermaxilary disjunction-A three-dimensional FEM study of a human skull. Journal Of Biomechanics. 2008;41:376-382.

18. Baldawa R S, Bhad W A. Stress distribution analysis during an intermaxillary dysjunction: A 3-D FEM study of an adult human skull. Annals of Maxillofacial Surgery. 2011 JanuaryJune; 1(1)

19. Provatidis C.G., Georgiopoulos B, Kotinas A, McDonald J.P. Evaluation of craniofacial effects during rapid maxillary expansión throuhg combined in vivo/in vitro and finite element studies. European Journal of Orthodontics 2008;30:437-448.

20. Wang D, Cheng L, Wang C, Qian Y, Pan X. Biomechanical analysis of rapid maxillary expansion in the UCLP patient. Medical Engineering \& Physics 2009;31:409-417.

21. Gautam Pawan, Zhao Linping, Patel Pravin. Biomechanical resposte of the maxillofacial skeleton to transpalatal orthopedic force in a unilateral palatal cleft. Angle Orthodontist, 2011;81(3)

22. Gautam Pawam, Zhao Linping, Patel Pravin. Determining the osteotomy pattern in surgical assisted rapid maxillary expansion in a unilateral palatal cleft. A finite element model approach. Angle Orthodontist, 2011;81(3)

23. Lagravere M, Carey J, Heo G, Toogood R, Major P. Transverse, vertical, and anteroposterior changes from bone-anchored maxillary expansion vs traditional rapid maxillary expansion: A randomized Clinical trial. Am. J. Orthod. Dentofacial Orthop 2010;137:304.e1-304.e12. 